\title{
Supporting Documentation and Evaluation of Student Progress in Digital Fabrication
}

\author{
Nasrin Aktera, Nijar Hossainª, Iván Sánchez Milaraª, Georgi V. Georgieva* \\ a Center for Ubiquitous Computing, University of Oulu, Finland \\ *Corresponding author e-mail: georgi.georgiev@oulu.fi
}

\begin{abstract}
:
Documentation is a common practice that constitutes an integral part of the design process in design education; for example, setting out the details of the fabrication process while designing a prototype in a digital fabrication laboratory (Fab Lab). In this article, we introduce a documenting application called Protobooth Oulu, with different features for documenting and evaluating student work. The application allows remote and distributed work on digital fabrication projects. This tool adheres to existing guidelines for Fab Lab users in creating, managing, and updating personal or group projects. Based on the submission of the students' weekly documentation, this application implements questionnaires for evaluation and focuses on a strategy of giving timely feedback to students in the context of digital fabrication education in Fab Lab. The ultimate objective of this tool is to enable educators to provide meaningful feedback that is visible to both the instructor and students.
\end{abstract}

Keywords: documentation; design process; feedback; digital fabrication

\section{Introduction}

Iterative prototyping is an essential instrument in design thinking (Leifer \& Steinert, 2011).

Prototyping and materialisation are instrumental in achieving a high level of creativity in design outcomes (Georgiev \& Taura, 2015). The design process might sometimes lead to difficulties and time-consuming steps due to the processes of rethinking, reconstructing, and rebuilding to develop a new prototype. Documentation facilitates these processes during prototyping but also gives rise to new challenges in designing prototypes.

Members of the digital fabrication community usually share their common interests in the creation of new devices, artefacts, and objects (Cautela et al., 2014; Milara et al., 2019; Stager, 2013). For example, each contribution in digital fabrication laboratories (Fab Labs) creates numerous opportunities by combining art based on engineering with the design and development of a modern artefact (Stager, 2013). To attain an in-depth understanding and produce a smooth design, regardless of the type of prototype involved, different solutions have been proposed to facilitate the documentation process (Adeegbe, 2019; Milara et al., 2019). Such tools help students capture and share their prototype designs within a team and with an instructor, and with such tools, the early 
stages of prototyping are made easier and are better documented for future use (Adeegbe, 2019; Barhoush et al., 2019; Milara et al., 2017; Sjöman et al., 2017; Soomro et al., 2021a).

The present study focuses on the roles of instructors and students in facilitating documentation and customising feedback using a dedicated documentation tool. In this paper, we introduce an application (app) called ProtoBooth Oulu, which supports multiple features for documentation in design education. The app is particularly suitable for remote and distributed work by students and teams on digital fabrication projects. The app is inspired by design education in the Fab Lab context and supports interactions between students and instructors while prototyping and documenting in Fab Lab. Using the app, instructors were given the ability to set up evaluation and feedback parameters and were able to evaluate the documentation and prototyping of the students using module app as an individual or as a group. The instructors' feedback incorporates several features, such as different stages of evaluation and rating (e.g., Likert scales, semantic differential [SD] scales, direct grading). As a result, students can view their evaluation in detail based on specific criteria related to the actual work in the form of feedback from their instructors. We present a unique approach for an environment where instructors and students can interact by documenting the design process and providing feedback as evaluation in Fab Lab or other design education contexts.

\section{Background Work}

Several tools are used to solve different design problems in Fab Lab. A recent tool, Document while Doing, allows documentation of the design and prototyping techniques (Milara et al., 2019). Document while Doing comprises an alternative solution to the existing problem of Fab Lab documentation on the network. The key reason for its use is simplicity, and it consists of three essential elements: 1) mobile app, 2) server, and 3) web aThis app allows the collection of pictures with notes (Milara et al., 2019), and supports voice messaging. It can manage and store data on a back-end server and display it on a website. Later, the content of the site can be changed or edited on the web. To reflect the process-oriented approach in documentation, Document while Doing is a software tool whose main task is reporting (Milara et al., 2019).

Maker and do-it-yourself communities share documentation, such as videos, pictures, or conceptual sketches. Conducting proper documentation requires adherence to regulations and provision of descriptions with images so that any Fab Lab user can get a good grasp of the design procedure (Määttä \& Troxler, 2011). In this paper, we attempt to create a new technique with documentation tools to help users design their prototypes design education context in Fab Lab Oulu. Our app implements flexible feedback features focused on that context. Finally, we will perform usability testing to evaluate the system's performance for operational activities in Fab Lab Oulu.

\subsection{Fab Lab Documentation Process}

Various documentation methods can be adopted in Fab Lab operations. Some authors have presented two ways of conducting documentation (Tseng \& Tsai, 2015). The first approach is recipelike or tutorial-like documentation, which is done by stating the instructional guidelines and setting up some steps that users need to apply in their documentation. In the recipe-like approach, we need to determine and write the ingredients, and from the ingredients, we need to figure out the materials and tools for writing the documentation. The second method is the process-like or storylike approach. Here, the description must follow the guidelines (Milara et al., 2019). Users must perform each task, focus on the process, and then complete the next task in Fab Lab. Information 
about the problems encountered must be included, along with a brief description of the problemsolving strategies they employed.

Process-oriented documentation is more beneficial to the Fab Lab community and is quite helpful for future researchers. If a project does not work, or a user is stuck on a step that requires documentation for future researchers and the Fab Lab community, researchers or developers in Fab Lab may not have followed the complete documentation designed through the process-oriented approach. However, they will be able to view the answers to some research questions or figure out some similar concepts that might be needed to develop their projects.

\subsection{Fab Lab Documentation Tools and Platforms}

Fab Labs rely on networks and sharing principles for information about design (Soomro et al., 2021b). Open-source web platforms (e.g., thingiverse.com, instructables.com, etc.) are not sufficient to serve the needs of the Fab Lab network (Schoffelen \& Huybrechts, 2013). Various approaches for creating documentation focus on designing and prototyping in Fab Lab. One example is Proto Space, a website documentation tool with a Drupal platform that was conceptualised for creating documentation in Fab Lab (Oosterhuis, 2005). Another example is Swiss Fab Lab, whose platform is WordPress, and was also intended for making documentation in Fab Lab (Orfanou et al., 2015). Another example is the Fab Lab community tools website, fabfolk.com, which was created to elicit documentation elements from primary sources (Määttä \& Troxler, 2011).

\subsection{Research Goals}

This study aims to create a documentation tool for design education in the Fab Lab context and to provide a system that helps students design their artefacts. This system facilitates interactions between students and instructors and sustains the provision of feedback using the ProtoBooth Oulu aThe students are given a new way of viewing and submitting their weekly course assignments. Multiple interactions and shared content are some of the main features of the ProtoBooth Oulu aDesigning and documenting the creation of new prototypes using a storytelling approach is considered the best way of using this aThere is an individual dashboard for every student to see private comments and grading from the instructor's side, and various semantic differential scales (SD), Likert scales, and ratings are used for the prototype's design evaluation and giving feedback (Hamon et al., 2014). Teachers can provide feedback on the students' project, design, and documentation of the prototype. For example, teachers can give a rating of 1-5 based on task quality, and the Likert scale serves a series of questionnaires that help provide feedback on students' prototype design and documentation. Possibilities for different questions are provided, which can relate to the students' design of the prototype and their experience of doing their project.

Through this app, students can receive support in their weekly assignments and group projects by capturing and sharing content and interacting with their group members and instructors, thus supporting knowledge sharing process.

Additionally, they can utilise the generated knowledge based on the prototype design concepts and receive timely feedback while making the documentation and developing their prototypes. Further research aims to create another tool for the admin side through which the design researcher will be able to acquire data from the database and conduct analytical research on the design and prototyping process. 


\section{Method}

We developed a system app called ProtoBooth Oulu, which is intended to facilitate the communication of instructors and students in design education in Fab Lab. We formulated specific features, such as feedback that makes use of scales to evaluate the students' projects in Fab Lab, and these features support system tools for instructors' feedback evaluation. Our system app involves two specific software clients (students and instructors). Moreover, the server side stores all data and logic coming from the users, while the client side provides a design layout where users can input data for specific tasks. We used the following features in our application:

- $\quad$ Android mobile application

- $\quad$ Application server architecture

- $\quad$ Students' and instructors' interaction process

\subsection{Concept of the Students' Documentation System}

The architecture of the ProtoBooth Oulu app was designed to accommodate weekly work on projects that could be structured within a group. Figure 1 illustrates the concept of the students' documentation system.

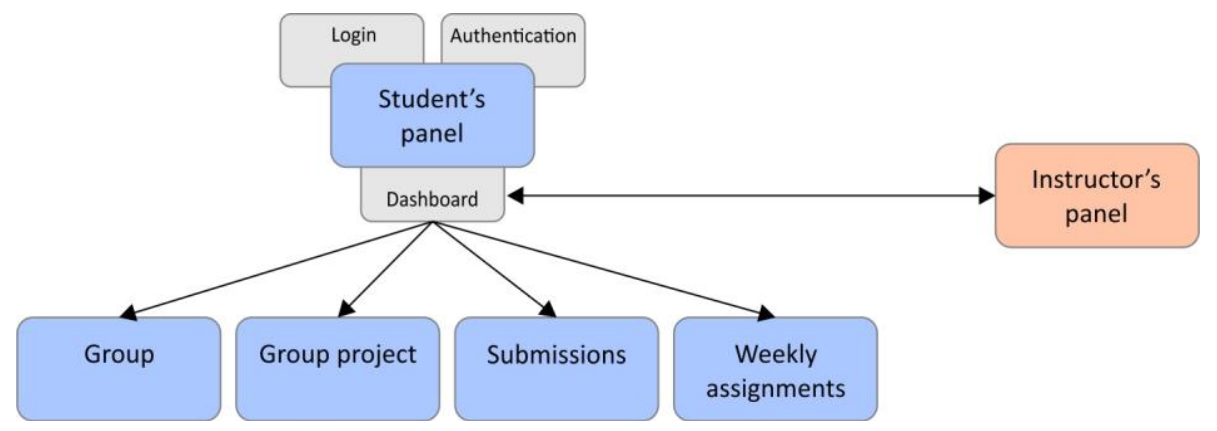

Figure 1: Flowchart of the students' module in the system

The app secures login and authentication for every student. The weekly projects unit is where submitting and assigning weekly exercises for individual students are undertaken. Designing and developing the group project through the unique dashboard for scaling, capturing, establishing multiple interactions within a group, submitting the final project, and giving feedback are the core features of this aAdditionally, private feedback and scaling (e.g., SD scales, Likert scales, ratings, etc.) are visible on this homepage through an individual student dashboard.

\subsection{Concept of the Instructors' Evaluation System}

Fab Lab provides a unique concept and develops individual creativity in different artefacts with the help of the instructor's evaluations of the students' work. For this reason, we designed an instructor's feedback system for the students' documentation throughout the design process. 


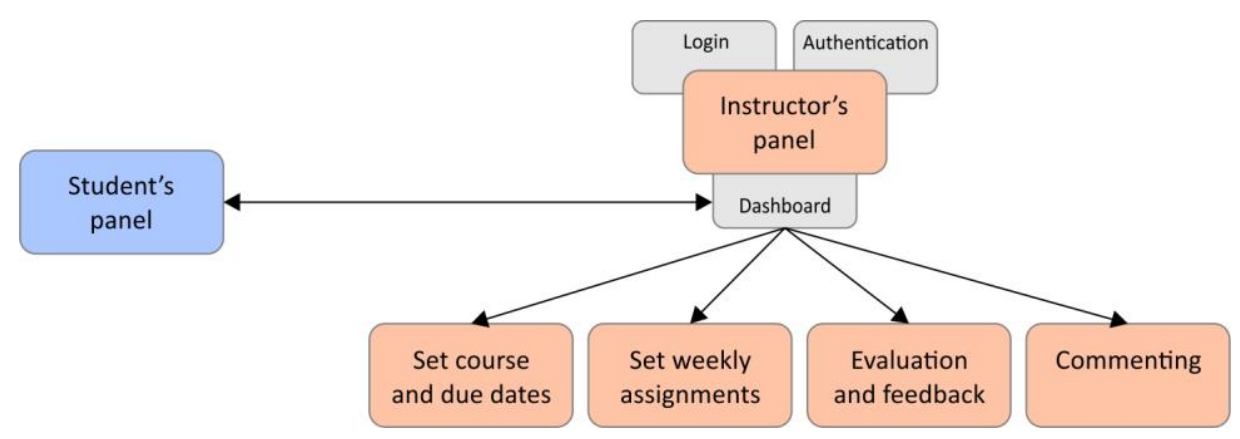

Figure 2: Flowchart of the instructors' feedback in the system

Figure 2 shows the possibility of creating a course environment and providing instructor feedback for the students' prototypes. Based on the above design, instructors can interact with students through this system. We used multiple customisable features for the instructor's feedback as an evaluation of the students' work.

\section{Developed Tool}

\subsection{Feedback and Interaction}

Each student's project is shown on the dashboard with the title, description, and date. The private comments button is a useful feature for receiving timely feedback from the instructor (Figure 3 ).

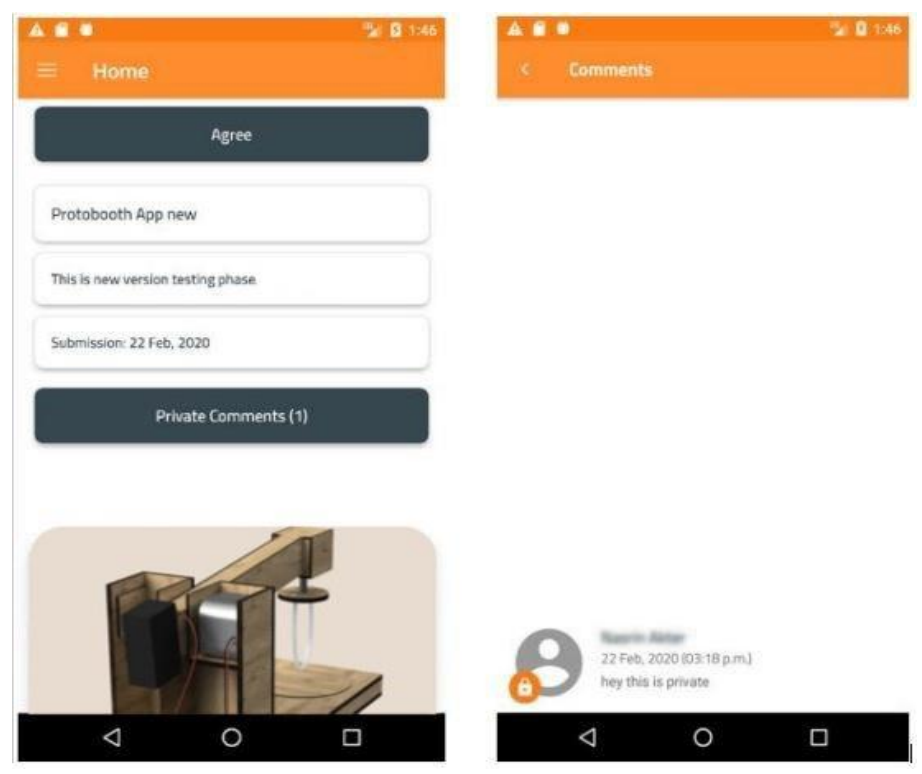

Figure 3: Feedback and interaction on the home screen (left) and private comment (right)

Receiving timely feedback from the instructor can help students learn more about the current stage of the designed prototype within a group or as an individual. It also enables students to accumulate experience and knowledge about the design. Each of the groups gets an individual dashboard based on the instructor's feedback and may include evaluations using customisable scales and ratings on the project stages, focusing on the students' design process, technique, and creativity. 


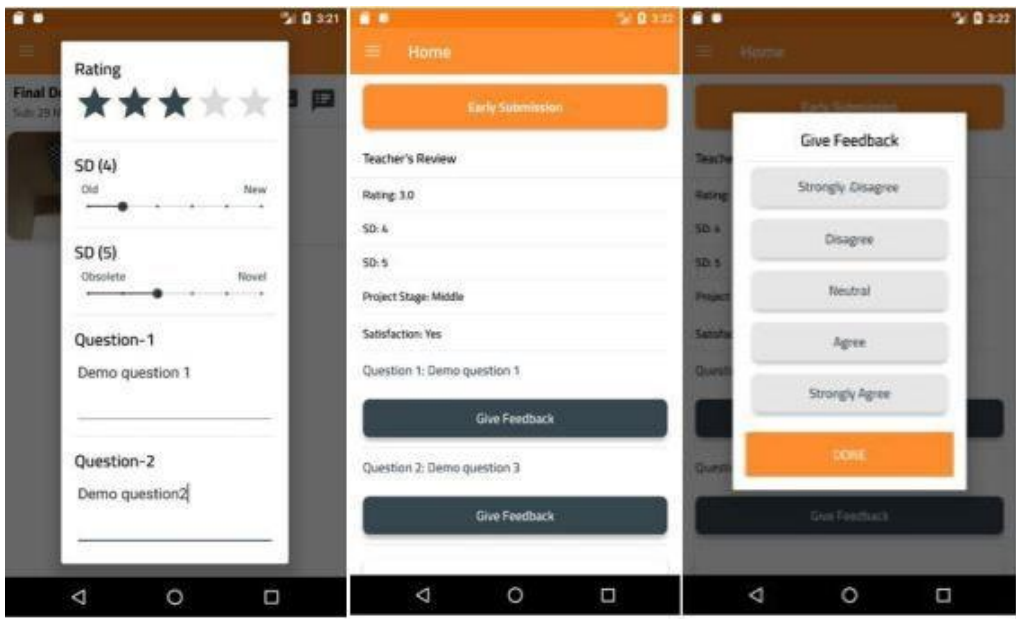

Figure 4: Notifications of each scaling from the instructor: scales and questions (left); overview (middle); and response (right)

Figure 4 shows an example of the instructor's feedback that is visible on the students' home page. The students are also able to view their current submission of the final project and answer questions. There are about 90 students in the class; usually, each group consists of 3-4 students. In this unit, every group member will interact with one another and update the title, description, and images as per requirements. Typically, the students have 7-8 weeks to finalize their group projects with documentation delivered at initial ideation, weekly development, and final project stages.

\subsection{Individual Weekly Tasks}

The students need to navigate the weekly project section and select the weeks they want to choose for their assignments before assigning individual tasks. Sharing and multiple interaction features are not available in this section. The current submission of each project is visible after the submission of each assignment.

As shown in Figure 5, each student needs to navigate and open the available weekly tasks from the weekly view section. After viewing the task, the student is able to capture the prototype design, document the whole process, and give the assignment a title. After successfully submitting the task, the student can view the submission data in the history navigation section.

\subsection{Instructors' Setup and Feedback}

The instructor is able to create and update their own profile after completing the domain email verification. They can also look at each student's specific task, such as sketch prototype, prototyping design implementation, and documenting from the instructor's dashboard. After receiving the assignment from the student's panel, the instructor has the ability to modify or delete the student's assignment from their board, as shown in Figure 6.

Students can look up the submission due date assigned by the instructor, and the instructor can receive the students' tasks as early or late submissions with the due date postmarked. This feature often plays a significant role in interactions between students and educators. 

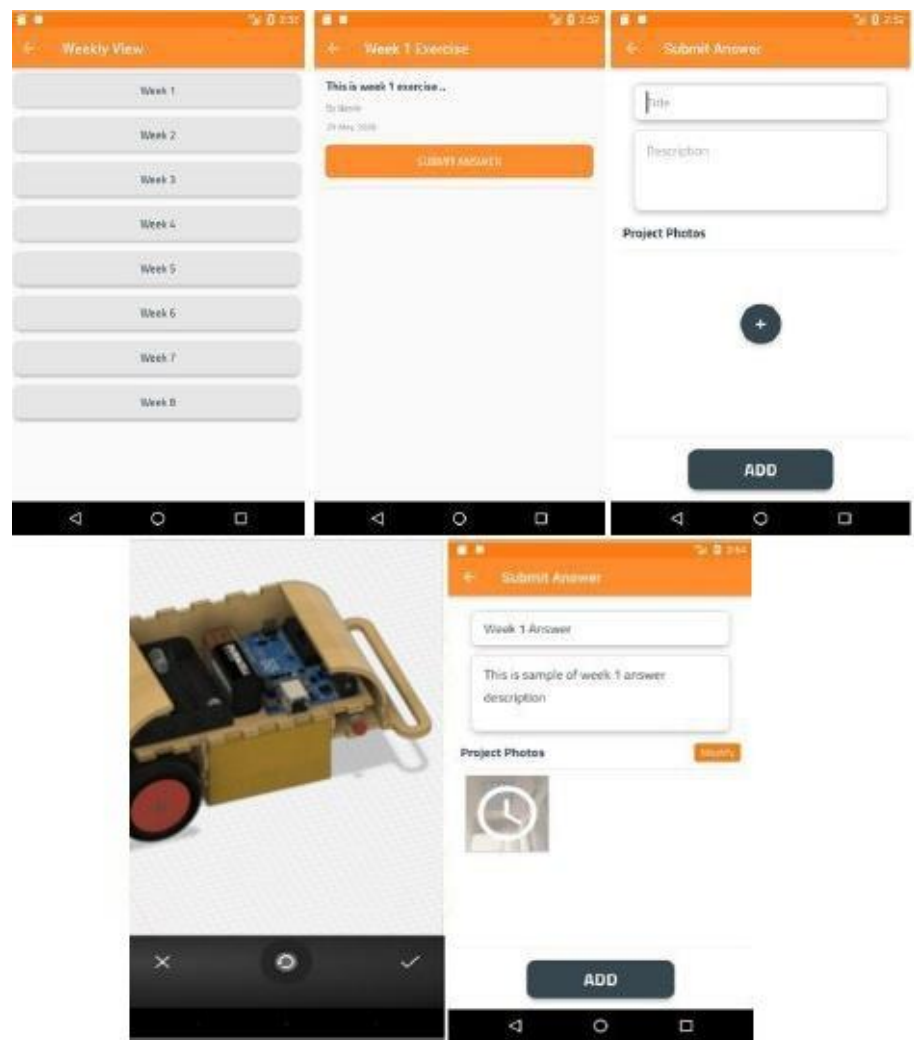

Figure 5: Individual weekly task (top images) and capturing prototype (bottom images)

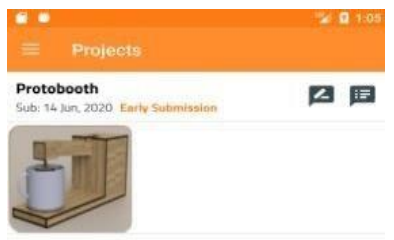

Figure 6: Instructor's board

\subsection{Instructor's Feedback on the Current State of Individual Projects}

Students can enquire about the process and determine how to implement the prototype to ensure the quality and uniqueness of their work. The students receive notifications about their project status on a weekly basis through the dashboard, which is shown in Figure 7. The instructor can view each project along with the submission deadline. The instructor can also modify items using the add or delete functions. 

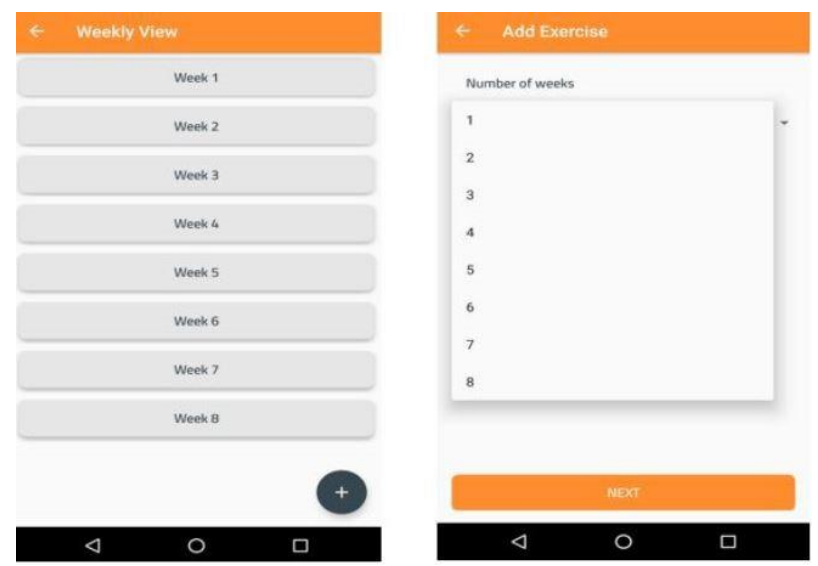

Figure 7: Weekly task lookup of the students' prototypes: weekly view (left) and adding weeks (right)

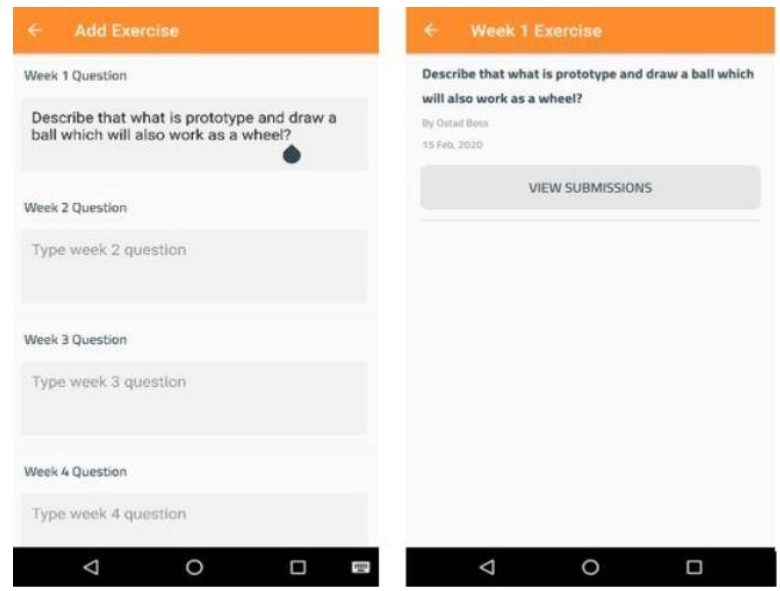

Figure 8: The weekly questions assigned by instructors: inputting questions (left) and viewing answers (right)

Figure 8 demonstrates that this practice enables students to access project creation and individual achievements. Each report has a view icon that allows students to view the questions and create new projects for another weekly report.

\subsection{Scales}

Assigning different SD scales is possible with this application. Figure 9 shows an example of the scales old-new and obsolete-novel, which provide an opportunity to offer feedback on aspects such as creativity. For example, if the newly designed prototypes are completely unique and creative, then they are marked as novel based on the newness and creativity of the project.

\subsection{Project Stage Evaluation}

The system also allows evaluations of project stages, for example, in the early, middle, and final stages, as shown in Figure 10. The instructor is responsible for creating a specific task, assigning the deadline, and using a scaling rating to grade the students' projects. Therefore, instructor can monitor the period based on whether a student's submission task is in the early, middle, or final stages. 


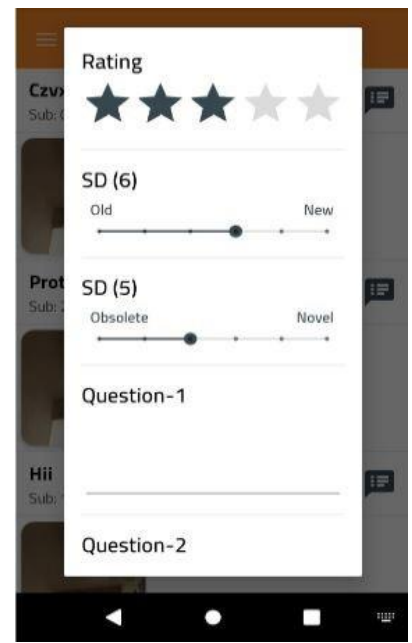

Figure 9: Feedback through ratings, semantic differential scales, and questions

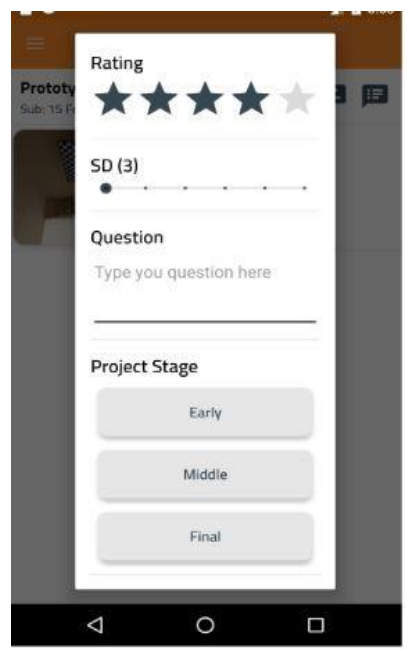

Figure 10: The project stages

\subsection{Final Stage Evaluation}

Students can view their evaluation in the feedback system, which comes from the instructor's module. After the design and documentation of the prototype has been submitted from the students' module, the instructor can evaluate the projects. Students can view the results of the proposed projects on their dashboard. Figure 11 shows the feedback system, which is beneficial for decision making. The characteristics are as follows:

- Instructors can share their feedback through the mobile application, which means that less time is spent on the task.

- Instructors can make timely decisions while students are making the prototype design.

- This feedback feature can be viewed privately by the instructor and the students. 


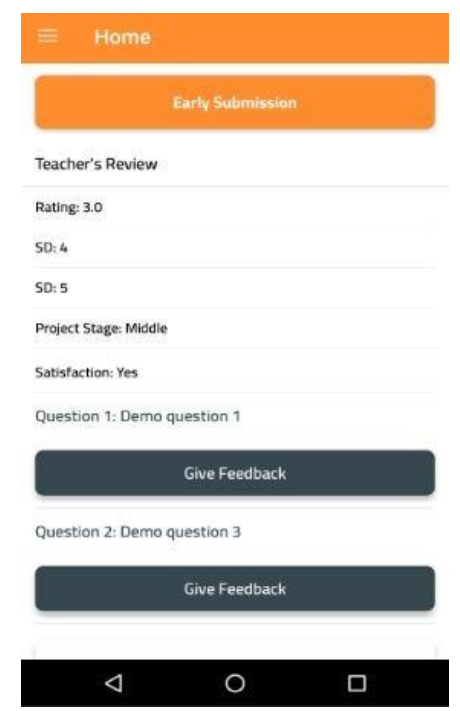

Figure 11: Evaluation view

\subsection{Usability Evaluation and Limitations}

We performed functional testing while developing the app and tried to make it user-friendly. Furthermore, we conducted a basic usability evaluation of the system to allow us to gather instructors' and students' feedback and compare it through the system. For this research, nine individuals, all students, aged between 22 and 36 years participated. Participation was voluntary, and they were informed of the concept underlying the application. The participants acted as instructors or students to complete the testing part of the form. We then captured the test case scenarios for the analysis and evaluation of the results. We used System Usability Scale (SUS) questionnaire. The SUS provides a tool for measuring usability (Lewis \& Sauro, 2009; Orfanou et al., 2015). We obtained SUS scores of 72.14 and 71.07 for the instructor and student participants, respectively. Both modules performed well (above 70) in the evaluation phase in terms of the SUS score, and as the student and instructor scores were similar, this indicates that both sides will perform well during actual utilisation.

There are limitations of our approach. This app needs further tests for performance enhancement. A limitation is that currently the app can run only on Android devices. Moreover, the design of the app is taking into account current practices in Fab Lab Oulu and needs further testing in other digital fabrication education contexts.

\section{Conclusion}

The proposed tool supports documenting and assigning specific feedback to the students' prototype design and documentation, particularly in remote and distributed work on digital fabrication projects. We conducted this research to make the process of providing feedback more flexible for instructors so they can view their students' work with less time and provide feedback quickly. This system has features such as a satisfactory level and project stage, which are essential functions that facilitate decision making.

Fab Labs needs a documentation tool for writing down the challenges and obstacles faced during the early design stages of prototypes that is easily accessible. Through this application, instructors give their evaluation of projects on customizable criteria, while students share their overall feedback and comments through the system. Based on the students' remarks, it was evident that they were able to 
learn more about their design process and project stages through the feedback. The questions and answers feature provided in this system is also helpful for students. The development and utilization of a shared process documentation and evaluation app can be very useful in remote learning environments, especially in the context of design and engineering for fabrication.

\section{References}

Adeegbe, J. M. (2019). A System Supporting Analysis of Prototyping in Fab Lab Education. Master thesis, University of Oulu, Oulu, Finland.

Barhoush, Y. A., Erichsen, J. F., Sjöman, H., Georgiev, G. V., \& Steinert, M. (2019). Capturing prototype progress in digital fabrication education. Proceedings of the Design Society: International Conference on Engineering Design, 1(1), 469-478.

Cautela, C., Pisano, P., \& Pironti, M. (2014). The emergence of new networked business models from technology innovation: an analysis of 3-D printing design enterprises. International Entrepreneurship and Management Journal, 10, 487-501.

Georgiev, G. V., \& Taura, T. (2015). Using idea materialization to enhance design creativity. In: DS 808 Proceedings of the 20th International Conference on Engineering Design (ICED 15) Vol 8: Innovation and Creativity, Milan, Italy, 27-30.07.15, 349-358.

Hamon, C. L., Green, M. G., Dunlap, B., Camburn, B. A., Crawford, R. H., \& Jensen, D. D. (2014). Virtual or physical prototypes development and testing of a prototyping planning tool. Tech. rep., United States Air Force Academy Air Force Academy United States.

Leifer, L. J., \& Steinert, M. (2011). Dancing with ambiguity: Causality behavior, design thinking, and triple-loop-learning. Information Knowledge Systems Management, 10, 151-173.

Lewis, J. R., \& Sauro, J. (2009). The factor structure of the system usability scale. In International conference on human centered design (94-103). Springer, Berlin, Heidelberg.

Määttä, A. \& Troxler, P. (2011). Developing open \& distributed tools for fab lab project documentation. In: OKCon.

Milara, I. S., Georgiev, G. V., Riekki, J., Ylioja, J., \& Pyykkönen, M. (2017). Human and Technological Dimensions of Making in FabLab. The Design Journal, 20(sup 1), S1080-S1092.

Milara I. S., Georgiev G. V., Ylioja J., Özüduru O., \& Riekki J. (2019). "Document-while-doing": A documentation tool for fab lab environments. The Design Journal 22(sup 1), S2019-2030.

Oosterhuis, K. (2005). Protospace 2.0. In: Disappearing Architecture, Springer, 218-235.

Orfanou, K., Tselios N., \& Katsanos, C. (2015). Perceived usability evaluation of learning management systems: Empirical evaluation of the system usability scale. The International Review of Research in Open and Distributed Learning, 16, 227-246.

Schoffelen, J., \& Huybrechts, L. (2013). Sharing is caring. sharing and documenting complex participatory projects to enable generative participation. Interaction Design and Architecture 18 (IxD\&A 18), 9-22.

Sjöman, H., Erichsen, J. A. B., Welo, T., \& Steinert, M. (2017). Effortless capture of design output a prerequisite for building a design repository with quantified design output. 2017 International Conference on Engineering, Technology and Innovation (ICE/ITMC), 564-570. 
Soomro, S. A., Barhoush, Y. A. M., Gong, Z., Kostakos, P., \& Georgiev, G. V. (2021a). Tools for recording prototyping activities and quantifying corresponding documentation in the early stages of product development. Proceedings of the Design Society, 1, 3159-3168.

Soomro, S. A., Casakin, H., \& Georgiev, G. V. (2021b). Sustainable design and prototyping using digital fabrication tools for education. Sustainability, 13(3), 1196.

Stager, G. S. (2013). Papert's prison Fab Lab: implications for the maker movement and education design. In: Proceedings of the 12th international conference on interaction design and children, 487490.

Tseng, T., \& Tsai, G. (2015). Process products: Capturing design iteration with digital fabrication. In: Proceedings of the Ninth International Conference on Tangible, Embedded, and Embodied Interaction, 631-636.

Author Bios:

Nasin Akter completed MSc at the Center for Ubiquitous Computing, University of Oulu, Finland focusing on the instructors' feedback in digital fabrication.

Nijar Hossain completed MSc at the Center for Ubiquitous Computing, University of Oulu, Finland on the topic of personal and team documentation in education context.

Iván Sánchez Milara is a PhD student and teacher at University of Oulu, Finland, and instructor at FabLab Oulu. His research focus in integrating digital fabrication into formal education. His research interests also include $\mathrm{HCl}$, digital fabrication and learning technologies.

Georgi V. Georgiev is an Associate Professor at the Center for Ubiquitous Computing, University of Oulu, Finland. His research interests are design creativity, idea generation, design cognition, digital fabrication and prototyping, design thinking, user interaction, and user experience.

Acknowledgements: This study has been partially financially supported by Academy of Finland 6Genesis Flagship (grant 318927) and by the Erasmus+ project "Bridging the creativity gap" (agreement number 2020-1-UK-01-KA202-079124). 